\title{
Distribution System Restoration Based On Optimized Heuristic Search Method
}

\author{
Akba Zoheer Mohammed \\ Director of Education in Al-Anbar \\ Anbar-Iraq \\ Okbah80@gmail.com
}

Article History: Received:11 January 2021; Accepted: 27 February 2021; Published online: 5 April 2021

\begin{abstract}
Service recovery is still among the most essential approaches to enhance the durability of their contemporary distribution system. Following the error location is identified and isolated; a correct SR program ought to be ascertained to resupply out-of-service places. 2 heuristic approaches are suggested to locate an efficient and speedy solution in contemporary power supply systems. For resolving the support recovery issue in distribution systems, change selection indices sectionalizes switch stated by an analytic plan in addition to a practicable optimized heuristic graph-based process are given. The formulation of the issue includes four different functions like optimizing the complete load restored and cutting back the amount of changing operations. Maximizing the best priority restored loading, also decreasing load decreasing. A nice evaluation of change indices is used for many player tie sticks from the apparatus to think about the ideal solution and minimize the complete quantity of shifting operations. A brand new graph-based program may be used for hunting the best sectionalizes change and diminishing the voltage fall. The precision as well as the validity of this process are analyzed in two regular electrical supply procedures. The outcomes of these suggested methods are utilized for IEEE regular bus test instance.
\end{abstract}

\section{INTRODUCTION}

In every contemporary electric supply system, raising reliability is thought of one of the most essential activities for electric distribution businesses. Together with the substantial expansion of contemporary power supply networks on earth, the chance of this fault happening from the system and also the consequent blackout for a couple of regions are raised. In order have the ability to keep the client's pride and also to increase network visibility, and then your fast recovery of this energy Supply to outside of support un-faulted area is necessary. As a result of this large number of switches, both branches and feeders in normal supply processes, it is not simple to rekindle a from support location entirely based upon the previous experiences of operators. Thus, with the dawn of computers, advances in engineering together with incorporation of energy technology, diminishing the outside of service areas is possible. Reaching quick service recovery, decreasing operators' errors in addition to impact raising network reliability and client's pride would be the vital benefits of recovery in the wise grid. In reality, the support recovery difficulty is to obtain the perfect setup by transferring the piles out of out of support un-faulted areas to neighborhood feeders after malfunction incident. In many single service recovery programs, there remain several problems to be critically contemplated. The most necessary objective of support recovery program is to earn the most of the safety of from support a lot that will have to be restored. Service recovery is accomplished by altering switch necessity; therefore the time scale required by the service recovery is dependent upon the amount of altering surgeries. Therefore, the number of altering operations has to be exploited to become in the minimum possible rate. In support recovery strategy it has to be considered that the distribution has to be restored into the customers of priority. The most significant limitation in the majority of distribution procedure is its own vertical construction as a consequence of many causes, like ease of discovering the error put error isolation and only misuse of apparatus. During the whole period of the support recovery, if the arrangement by the machine varies, the structure limit has to be thought about. Bus voltage, line part and present loading also vary through the whole service recovery program; hence it's important if these constraints cross their operational limitations. Consumers' satisfaction is contingent on the disturbance frequency combined with the length. So, for finding a fast treatment, the restoration procedure run time has to be paid off.

In previous decades, the numerous approaches are signaled to find a remedy to this curing difficulty from other viewpoints. The heuristic process is beneficial to get rid of service recovery difficulty. A fresh heuristic graph-based program is recommended for solving the help healing issues in supply networks. Objective functions are mimicked with all the fuzzy group, together with an optimization issue was solved with a genetic algorithm. In the process includes the blurry multi criteria analysis coupled with the grey relational analysis. A recovery problem in supply methods using dispersed manufacturing (DG) may be put into place. The maximum power distribution variety of DGs was changed to some 0-1 Knapsack problems and used branch-and-interface remedy to fix the retrieval problem. A qualitative analysis framework is introduced to find standing retrieval plans with their performance 
indicators, in addition to the analytic hierarchy process-based fuzzy-grey program. Mathematical programming was introduced to reinstall the system un-faulted part of the system. In reliability evaluation of innovative distribution platform programs will be released. In support recovery together with loading curtailment of service clients via direct accessibility management is executed. At a fuzzy decision making approach must see precisely the most desired restoration strategy with consideration distinct operational facets. In fuzzy cause-effect methods are helpful to mimic the heuristic comprehension inference part of the recovery program; nevertheless, fuzzy method won't guarantee the ideal answer. In petrinet united employing a rule-based specialist system is helpful to execute the aid recovery application. Rear G-net inference mechanism together with operation principles is implemented collectively side the specialist system process is adopted.

A publication formula for service retrieval at unbalanced three phase supply systems has been introduced. Within this newspaper, two heuristic approaches have been indicated for running service retrieval plans from contemporary supply systems. In this research function, an algorithm was designed based on heuristic search process with depthfirst investigation and breadth-first research methods to revive the supply procedure subsequent to the prevalence of lone fault and dual mistake. The restoration is done in order to lower the sum of shifting operations and to take advantage of the energy revived. The aim of the manner is to reduce the search area whilst resolving the matter. The machine after recovery should keep radial construction.

\section{LITERATURE REVIEW}

Several researches on utilities expertise indicate that client satisfaction is closely related to support interruption frequency and length. Service disruption length could be significantly diminished via successful service recovery processes. Speedy service recovery has multi-fold advantages [30]. By way of instance, it lessens the annoyance and the price of the outage for clients; it empowers the utility to restart generating revenue for energy revenue; it empowers the utility to offer improved support to priority clients like hospitals, police stations and fire departments, clients who might have contracts with all an utility for dependable electricity delivery, etc., [Wen-Hui Chen, Men_Shen Tsai]. The support recovery processes are utilized to reestablish just as much burden as possible by moving de-energized heaps from the out-of-service regions via system reconfiguration to additional encouraging feeders without breaking up electrical and operation constraints. In training, supply dispatchers will need to restore support to the recliner places as swiftly as possible using a minimum number of shifting operations. A minimum number of shifting operations is needed due to change life expectancy issues and labor constraints, because not all of switches in the community are now automatic [K. Miu, H. D. Chiang].

Increasing vulnerability of electricity methods to extensive blackouts is also a result of thicker system loading and current changes in business structure. The effect of prolonged blackouts on the general public, on the market, and about the grid itself makes quick effective recovery really significant. A successful system recovery plan lowers the effect of an air on clients and on the market of the affected region whilst decreasing the likelihood of harm to gear [M. Adibi, L. H. Fink]. The issue of power system recovery after a partial or complete blackout was studied for several years with particular focus on creating successful restoration strategies. Reactive energy imbalance, lively power imbalance and error of control and protection systems are a few of the principal issues the machine operators confront during recovery [N.A. Fountas, N.D. Hatziargyiou]. The responsive power imbalance is a result of big MVAr line charging of long transmission lines.

The actual energy imbalance is just big frequency deviation where the system stinks. To avert this actual power imbalance lots have to be picked up in little increments. Although blackouts occur infrequently, it's vital for an electrical utility to set up orderly restoration processes for such occasions and to concentrate on training and teaching both the shipment and engineer's operators. Service restoration programs have to help distribution employees in producing timely decisions to renew as much burden as possible at the out-of-service places. Service restoration also needs the utmost attention of team and public security problems. A succession of viable control operations to accomplish a last service recovery strategy has to be supplied.

\section{OPTIMIZED HEURISTIC SEARCH ALGORITHM}

Generally after recovery the consequent feeders must stay radial, with no violations of division voltage and loading limitations. Thus the issue of locating the system for recovery with minimal loss and minimal amount of shifting operations is a really complex mixed-integer, non-linear optimization issue. In a sensible distribution platform, in case there really are still "s" variety of buttons, then 2 s switch combinations are possible and a lengthy computation time must ascertain the settings for recovery. Thus the issue could be solved with heuristic search procedures. Heuristic approaches don't guarantee for optimal answers, but they result in sub-optimal solutions which are technically okay. It let's cuts the amount of choices to be assessed in a manageable amount. The issue from heuristic search system is solved in a binary decision tree. The heuristic research is an overall search system using domain specific knowledge to direct the research. The research methods enable traversing the distance of possible system 
conditions whereas domain-specific comprehension is vital in limiting the dimensions of this decision tree. It is made up of range of parents and child noted. It's called as the tree, because every parent has 2 child nodes. In this 15 signifies a parent, both 2 and 5 would be the kids. The child node 5 can also be a parent using its child 1 and 3 . 15is the main node and therefore it does not have any parents.

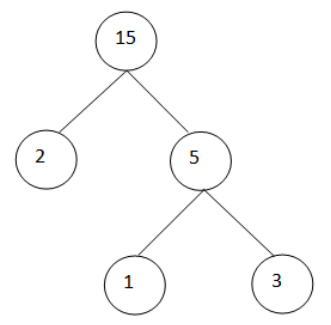

Figure 1: binary tree representation

The heuristic search methods are generally classified as i) General search ii) Depth-first search iii) Breadth-first search

General search: Generally search procedure, a binary tree has been shaped without imposing any restriction. Hence the memory area occupied by this procedure is much more.

Depth-first search: This search procedure investigates one route until its conclusion. If the route does not finish on the target condition, it will monitor and arbitrarily remain in a different route. It's extremely exact method, although the time necessary to acquire the best outcome is greater. A depth-first hunt [Gilles Brassard Paul Bratley] shrub is revealed in fig. 2. Depth-first search may be utilized to discover if a given directed graph is acyclic. It may likewise be employed to ascertain that a topological ordering of the nodes using a directed acyclic graph.

Breadth-first search: This search strategy assesses all of the choices concurrently, level by level. It's not a very exact method however, the time required to acquire the outcome is significantly less. Even a breadth-first search tree has been displayed in fig.3.3. Breadth first search is most frequently utilized to perform rigorous quest of an infinite graph, or to get the shortest route from 1 stage to another at a chart.
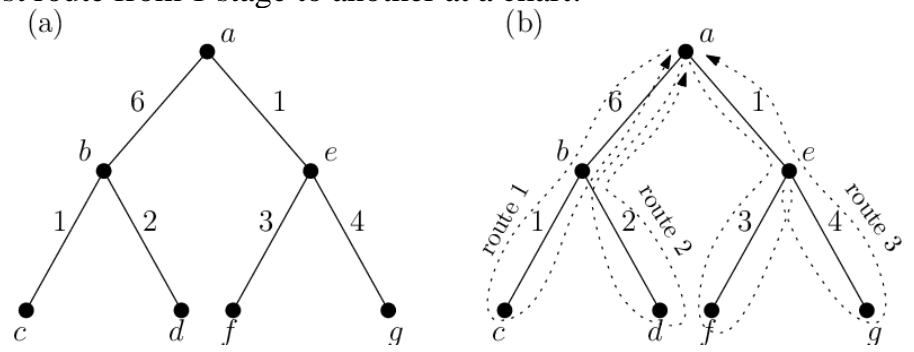

Figure 2: DFS

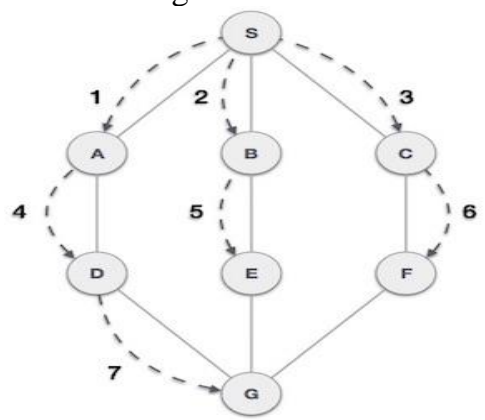

Figure 3: BFS

Service retrieval in distribution systems is devised as a multi-constraint with multi-objective difficulty, considering customer's obligation and load shedding execution. Four different target purposes can be considered: optimizing the Entire load restored, cutting the amount of changing surgeries, maximizing the ideal priority revived loading, along with also reducing load dropping. The Constraints would be the resulting radial system and minimal loss. The error on a single line and 2 distinct lines have been considered individually.

\section{Heuristic search method}


Considering that the distribution process has been modeled as a shrub, its different countries may be represented with a binary decision tree. Thus the issue is solved with heuristic search system. The binary choice factors and the choice vector for the machine are devised as follows: The conclusion, if the $\mathrm{j}^{\text {th }}$ change is opened or closed is obscured from the binary choice variable. $L_{j}=1$ (in case the $j^{\text {th }}$ change is shut) $=0$ (in case the $j^{\text {th }}$ change is started). The decision method is exemplified with a binary tree shown at fig.4. At the start of the procedure (root node), all of the decision factors are undeclared i.e., the first problem has unknowns. Since it's moved back on the shrub, the choice variables are pronounced either 1 or 0: at par "Id" of the shrub, it's (s-i) undeclared factors left. In the base of the tree (flat therefore) all of the choice variables are announced: every one of those $2 \mathrm{~s}$ nodes (leaves of the tree) are correlated with a potential solution (not always a viable one). An estimated representation of this tree is embraced by the principles used to create new nodes out of the present ones. So since the research method is performed, just part of this tree is built explicitly and nearly all of the trees need to be properly used. Domain specific knowledge can be utilized to prevent unnecessary research. At every node of each tree level, some undeclared factor is chosen to be announced next. After a variable is accepted, two nodes are made. These brand new nodes are known as successors.

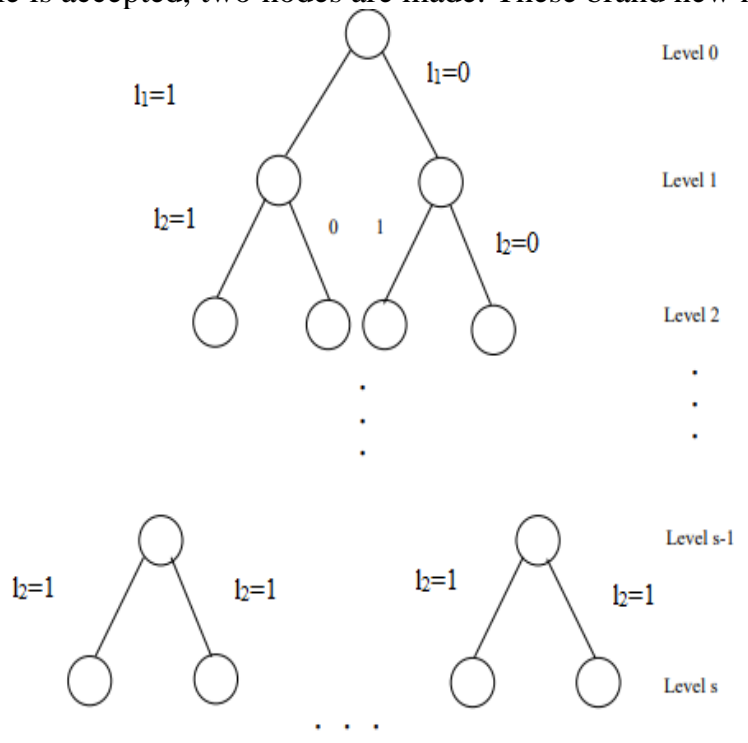

Figure 4: Decision tree representation

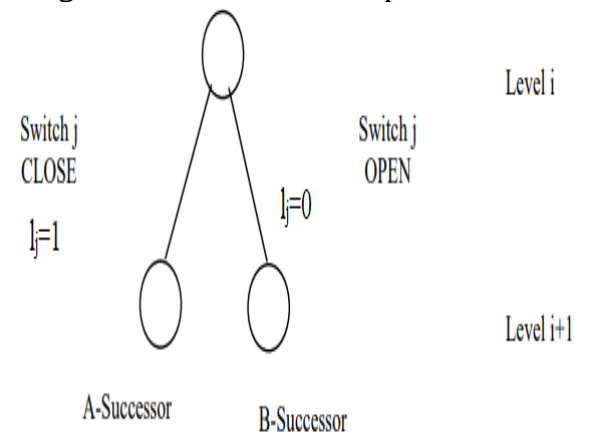

Figure 5: Decision tree successor

The option of the following variable to be announced depends on previous choices taken on a specific course; Practical principles, dependent on operator expertise, which are utilized to direct the research. Therefore, it's possible to mimic what a seasoned operator could do under the exact conditions. Additionally, to direct the search, functional rules may be used to purify the shrub, which might be significant in restricting the rise of the amount of choices to be considered.

\section{Search strategy}

The depth-first search system provides accurate results with bigger implementation time. The breadth-first search procedure provides less accurate results with minimal execution time. Hence, within this study operate a new algorithm is suggested for the research, which unites both depth-first and breadth-first research methods where the benefits of the methods have been attained. The breadth-first hunt is utilized to symbolize the job of this 
sectionalizing switches along with the depth-first search procedure provides the rankings of their tie buttons. While employing the depth-first investigation, it evaluates the prospect of shutting one tie button, so as to keep up the radial restriction. In the event the loads cannot be fulfilled, then it assesses the potential for shutting two tie buttons. The plan developed in this work assesses the prospect of closing only tie switch. When it isn't feasible, it hunts for the potential for shutting two tie buttons, three or even four tie buttons etc. The plan for the Step-by-step restoration process is provided as follows:

Step 1: The system information for the first setup is chosen and the working condition is assessed.

Step 2: In case the condition is normal, visit step1. If the Condition is unnatural, locate the faulted line and visit another step.

Step 3: Check for the availability of tie switches if available, go to the next step. If not, go to step 10.

Step 4: Assume closing of one tie switch, i.e., $n=1$, where " $n$ " is the number of tie switches to be operated.

Step 5: For tie switches from 1 to $t$, check for the radiality constraint for the available options where $t$ is the total number of tie switches.

Step 6: If any options that satisfy the radial constraint, go to step 8. If not, go to step 7 .

Step 7: Check for closing two tie switches, i.e., $n=n+1$, three tie switches, $n=n+2$, and so on and go to step 5 .

Step 8: If any option gives the min value of loss and its corresponding switching position by Run load flow and compute the loss for all the options.

Step 9: reveal result.

Step 10: There will be no power loss.

The flowchart of the aforementioned mentioned algorithm is displayed at fig.3. This algorithm is employed to lessen the amount of switching operations from the tie buttons. Similar process is employed to make the most of the energy revived with small alterations. Here the setup is assessed for greatest energy restored with a single tie button, i.e., recovery of loads. When it isn't feasible to revive all the heaps with one switch, yet another tie button is shut by opening an equal sectionalizing switch. So both tie-line and sectionalizing switches are worked. 


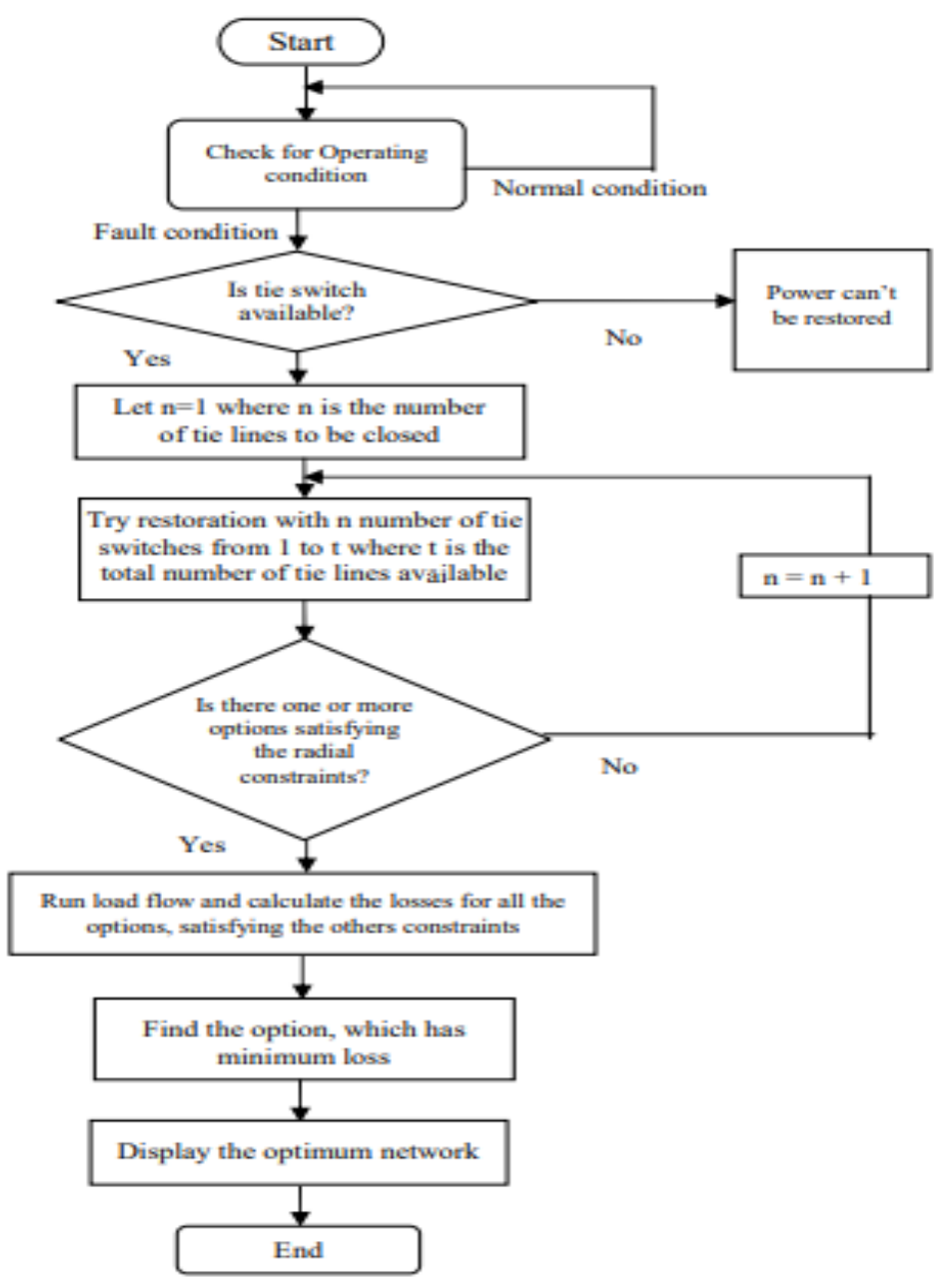

Figure 6: Flowchart representation for Heuristic search algorithm for restoration

This script employs the speedy load flow procedure for rapid service restoration functionality. Once an error happens from the supply process, the error lineup, either receiving or sending bus components are located. The adjoining lines and buses out of all probable directions are completely wended sequentially to clean out the error; the specific preliminary circuit breaker in these instructions is available and begins functioning. Hence, the feeder where the malfunction has happened and its own circuit breaker have already been discovered. For isolating the error, the adjoining lines and buses in each management are wended sequentially. The very first change in every management is located and begins working. Hence 3 areas are shaped by the system: Primarily, the out-of-service place is completely restored by activating circuit breaker of this feeder. Second, the damaged region has to be mended. Initially, the un-faulted place is moved into the neighboring claws based on the suggested algorithms. Before employing the procedure, we have to define change (TS and SS) applicants to the very first algorithm. All link buttons connected to the out-of-service un-faulted area would be the tie switch candidates to aid recovery execution. All of sectionalize switches into the out-of-service un-faulted area would be the sectionalize swap applicants. $\mathrm{H}$ jointly with $\mathrm{Z}_{\mathrm{path}}$ are obtained for each and every single participant TS. A weight variable is used to modify both these indices to an equal person indicator. The final indicator is clarified as:

$$
\mathrm{F}=\alpha \mathrm{Hi}+\beta \max \left(\mathrm{Z}_{\mathrm{path}}\right)
$$

Where $\alpha$ and $\beta$ are two weight factors $(0 \alpha<1$ and $0 \beta<1)$. The very first and the most indispensable index has greater weight factor when compared with another index. The number of weight factor could be initialized by PDN operators. The F listing is shaped in a growing sequence. 1 TS functions in compliance with the smallest load of function F (that the very first member of TS album), loading stream is guessed, and neighborhood limitations are appraised. If all system limitations are adequate, the recovery program is recognized and recovery procedure is stopped. When an overload or ion breach is present load shedding needs to work. After load losing execution, in the event the system limits are disappointing, another TS candidate has to be worked. 


\section{Load shedding}

For executing load-shedding from the town, quite nominal priority customers from out of service un-faulted area are well known. Even the SS is well understood that is only one of those hints mounted on non invasive customers in this field. The SS record is formed within an increasing structure in accordance with the amount of load shedding, and also the 1st candidate for load shedding can be well-known. The loading escape is put on the machine and also city limits have been assessed. If all system limitations are adequate, the recovery application is famous; differently, yet another load-shedding candidate has to be set into place. For executing loading falling from the machine, quite low priority customers from service un-faulted region are all recorded. The SS is recognized that is only one of those traces connected with non priority customers in this subject. The SS collection is shaped within a climbing sequence in accord with the amount of load shedding, and the very initial candidate to get load-shedding (minimal load shedding amount) is well recognized. Force stream calculation is place on the system as well as the system limitations are evaluated. If all of system constraints are satisfactory, the restoration program is recognized; otherwise, another load losing candidate needs to be placed into position.

\section{Next Tie Switch selection}

Assuming that one mistake has occurred in lines 58 from the 69-bus supply there's one direction from the setup which produces one loop out of the network. For eliminating this specific loop, the graph-based manner is used in order to think of the best SS from the loop administration. Hence the exact ideal sectionalizes switch is employed for reducing the voltage fall from the computer system. If all of system limits are sufficient, the restoration program is well known. In addition to from the 69-bus collectively sides 119-bus distribution strategies Shedding application has to be set into loop. If the system limits are disappointing following a load dropping is executed, the following TS and SS applicants will need to go worked.

Table 1 without load shedding Restoration results for 69-bus network

\begin{tabular}{|c|c|c|c|c|c|c|}
\hline Case & \multirow{2}{*}{$\begin{array}{c}\text { Fault } \\
\text { location }\end{array}$} & Fault Isolation & Restoration & \multicolumn{2}{|c|}{ Min. voltage } & \multirow{2}{*}{ Runtime (s) } \\
\cline { 4 - 6 } & & & & Bus & $\begin{array}{c}\text { Value } \\
\text { (p.u.) }\end{array}$ & \\
\hline 1 & $5-8$ & $5-8,8-9$ & $\begin{array}{c}12-44,51-60,10- \\
54\end{array}$ & 65 & 0.9000 & 1.03 \\
\hline 2 & $10-13$ & $\begin{array}{c}10-12,12-13, \\
12-67\end{array}$ & $48-13$ & 68 & 0.9000 & 0.48 \\
\hline 3 & $55-56$ & $55-56,56-57$ & $52-60$ & 68 & 0.9008 & 0.44 \\
\hline 4 & $18-20$ & $19-20,20-21$ & $14-22$ & 65 & 0.9012 & 0.38 \\
\hline
\end{tabular}

Table 2 Restoration results for 119-bus network without load shedding

\begin{tabular}{|c|c|c|c|c|c|c|}
\hline \multirow[t]{2}{*}{ Case } & \multirow{2}{*}{$\begin{array}{l}\text { Fault } \\
\text { Location }\end{array}$} & \multirow{2}{*}{$\begin{array}{l}\text { Switch fault } \\
\text { Isolation }\end{array}$} & \multirow[t]{2}{*}{ Switch Restoration } & \multicolumn{2}{|c|}{ Min. voltage } & \multirow[t]{2}{*}{ Runtime (s) } \\
\hline & & & & Bus & Value & \\
\hline 1 & $67-68$ & $67-68,68-69$ & $92-74$ & 75 & 0.9018 & 0.74 \\
\hline 2 & $32-33$ & $32-33,33-34$ & $\begin{array}{l}50-63,44-55,52- \\
53\end{array}$ & 35 & 0.9042 & 1.73 \\
\hline 3 & $104-105$ & $\begin{array}{l}104-105,105- \\
106\end{array}$ & $111-118$ & 106 & 0.9059 & 0.75 \\
\hline 4 & $90-91$ & 90-91, 91-92 & $\begin{array}{l}92-74,59-97,91- \\
96\end{array}$ & 76 & 0.9019 & 1.26 \\
\hline
\end{tabular}

That is revealed at Table 1, the alternative of SS relies upon the graph-based procedure. The minimal voltage of this network from the bus 65 is nearly 0.9 p.u. The runtime of this program for fault isolation and service recovery execution is $1.06 \mathrm{~s}$. For instance 2 in Table 1, 3 change operations are expected to isolate the error. Therefore, the fault location is dispersed in most directions. For instance 1 in Table 2, two swap operations are needed for isolating the error and also one change operation is necessary for service restoration execution. The minimum voltage at the bus 76 is nearly 0.9 p.u.

Table 3: Comparing results for different methods

\begin{tabular}{|c|c|c|}
\hline Method proposed by & Test case topology & Average runtime(seconds) \\
\hline Chao MH, Cheng TH & $\begin{array}{c}\text { Taiwan Power Company } \\
\text { distribution network }\end{array}$ & 12.04 \\
\hline Kumar Y, Das B & 32-bus & 330.42 \\
\cline { 2 - 3 } & 173-bus & 87.82 \\
\hline Khushalani S, Solanki J & Un-balanced 37-bus & \\
\hline
\end{tabular}




\begin{tabular}{|l|c|c|}
\multirow{2}{*}{ Proposed algorithms } & 69-bus & 0.58 \\
\cline { 2 - 3 } & 119-bus & 1.10 \\
\hline
\end{tabular}

Table 3 displays the typical software restoration execution. The applications runtime is diminished by suggested methods. And the outcomes of a few of the testimonials (assessing the runtime of support Runtime for many fault points from the system. According to the outcome of Table 3 shows the contrast between the outcomes of the suggested method The Main indices for enhancing the Support Restoration plan would be the recovery time and the amount of change operations.

\section{ADVANTAGES}

Reduce the technical weight of this operator to reestablish the network. The period needed to find the perfect outcome is not as due to the use of depth-first research and breadth-first lookup procedures. It may be applied to large scale programs too. Care is centered in locating the recovery process following the occurrence of an error on one line or two lines. It's likewise feasible for contemplating faults at greater quantity of lines concurrently.

\section{CONCLUSION}

An algorithm has been designed based on Heuristic Search strategy to supply system support recovery. The recovery issue is solved and examined in to two classes. The first class is employed to ascertain the setup with minimal number of shifting operations to renew the from service area after an error. The recovery is carried out by working just the tie buttons. The next category is employed to ascertain the settings for greatest quantity of electricity restored. The recovery is carried out by working both tie-line and sectionalizing switches. In the results, it's reasoned that the first class may render some heaps unenergized with minimal amount of switching operations and also the next group restores all of the affected lots with greater amount of switching operations and thus raises the price of shifting operation. Therefore depending on the area and need of recovery, any procedure can be chosen. The results demonstrate that these approaches may be used for resolving the most support recovery issues in modern supply networks.

\section{RECOMMENDATIONS FOR FUTURE STUDY}

In reduction it is $98.68 \%$ in voltage drop when the recovery is carried out with $43.13 \%$ and 98.81 percent with four shifting operations. It's concluded that the $20 \mathrm{MW}$ is installed at bus 9 from the 69 -Bus system there is a reduction of $30.23 \%$ Introduction of DG of appropriate dimensions and location reduces the system reduction and Recovery is also examined. It's seen from the results that when a DG of This DG following a fault online 2 the reduction in loss and voltage drop are all Voltage drop to a higher level.

Recommendations for study of best location and dimensions of single and Goals like minimization of recovery time, amount parts trashed, etc., and also the issue could be solved because of a multi-objective issue. The developed recovery algorithm may include many DG units' dependents on the requirement by means of a preparation approach. Restoration and reconfiguration could be produced for error analysis, contingency, power quality and stability advancement with DG units.

\section{REFERENCES}

1. C.Y. Teo, Wei shen, "Real- time generation of a dynamic restoration plan for bulk - power systems", Electric Power Systems Research, Vol. 53, 2000, pp.113-122.

2. "August 14 Blackout Significant Restoration Milestones", A report by Independent Electricity Market Operator (IMO), August 29, 2003.

3. D. Lindenmeyer, H.W. Dommel, and M.M. Adibi, "Power System Restoration -a bibiliographical survey", Electrical power and energy systems 23(2001), pp.219-227.

4. S. $\mathrm{Wu}, \mathrm{K} . \mathrm{L}$. Tomsovic and C.S. Chen, "A Heuristic Search approach feeder switching operations for overload, Faults, Unbalanced Floe and Maintenance", IEEE Transactions on Power Delivery, Vol.6, No.4, October 1991, pp.1579-1584.

5. Y.Y. Hsu, H.M. Huang, H.C. Kuo, S.K. Peng, C.W. Chang,K.J. Chang, H.S. Yu, C.E. Chow, and R.T. Kuo, "Distribution system service restoration using a heuristic search approach," IEEE Transactions on Power Delivery, Vol. 7, 1992, pp. 734-740.

6. L. Morelato and A. Monticelli, "Heuristic search approach to distribution system restoration", IEEE Transactions on Power Delivery, Vol. 4, No. 4, April 1989, pp.2234-2241.

7. Arturo S. Bretas, Member, IEEE, and Arun G. Phadke, Fellow, IEEE, "Artificial Neural Networks in Power System Restoration", IEEE Transaction on Power Delivery, Vol. 18, No. 4 Oct. 2003, pp.11811186. 
8. Joon-Ho Choi and Jae-Chul Kim, "Network Reconfiguration at the Power Distribution System with Dispersed Generations for Loss Reduction", IEEE Power Engineering Society Winter Meeting, 2000, Vol. 4, 23-27 Jan. 2000, pp.2363 - 2367.

9. M. Adibi, L. H. Fink, "Power system restoration planning", IEEE Transactions on Power System, Vol. 9, Issue : 1, Feb. 1994, pp. 22-28.

10. Qin Zhou, Dariush Shirmohammadi and W. Edwin Liu, "Distribution Feeder reconfiguration For Service Restoration and Load Balancing”, IEEE Transactions on Power Systems, Vol. 12, No. 2, May 1997, pp. 724-729.

11. T. Nagata, H. Sasaki, and M. Kitagawa, " A method of determining target configuration for power system configuration for power system restoration by means of mixed integer programming approach", Trans.IEE Japan, Vol.114-B, No.2,1994.

12. T. Nagata, S. Hatakeyama, M. Yasuoka and H. Sasaki, "An Efficient Method for Power Distribution System Restoration Based on Mathematical Programming and Operation Strategy", International Conference on Power System Technology, Powercon 2000, IEEE, Vol. 3, 4-7, Dec 2000, pp.1545-1550.

13. Yogendra Kumar, Biswarup Das, and Jaydev Sharma, "Service restoration in distribution system using non-nominated sorting genetic algorithm”, Electric Power System Research, 2005.10.008, pp.1-10.

14. Enrico Carpaneto, Gianfranco Chicco and Emiliano Roggero, "Comparing deterministic and simulated annealing based algorithm for minimum losses reconfiguration of large distribution systems".0-78037967-5/03/2003, IEEE.

15. Yoshikazu Fukuyama and Hsaio-Dong Chiang, "A parallel genetic algorithm for service restoration in electric power distribution systems", Proceedings of IEEE International Conference on on Fuzzy Systems, Vol. 1, 20-24, March 1995, pp. 275-282.

16. K. Miu, H. D. Chiang, B. Yuan, and G. Darling, "Fast service restoration for large-scale distribution systems with priority customers and constraints," IEEE Transactions on Power Systems, Vol. 13, No. 3, Aug. 1998, pp. 789-95. 